\title{
To the question of coordinate system choice within composition of the spatial generalized MIMO radar ambiguity function
}

\author{
Rodion Vodolazov, Valery Chapursky", and Andrey Filatov \\ Bauman Moscow State Technical University 5, 2nd Baumanskaya Str., 105005, Moscow, Russia
}

\begin{abstract}
The issue of the choice of spatial coordinate system which is suitable to physical sense of the radar concept is explored on the basis of the derivation of spatial generalized ambiguity function for MIMO antenna system and of its cross-section analysis. The comparison results of generalized ambiguity function cross-sections for two dimensional target coordinates in Cartesian, spherical and modified spherical coordinate systems are given for orthogonal LFM waveforms radiated by the elements of linear MIMO antenna system transmit. This proves the desirable choice between coordinate systems with regard of physical sense of the current task.
\end{abstract}

\section{Introduction}

The definition of generalized ambiguity function in spatial coordinates is logically used during spatial resolution performance of radar, sonar and remote sensing systems based on MIMO up to date principles (see [1, 2], for example). The issue of generalized ambiguity function coordinate cross-sections for various antennas system configurations and collections of transmit waveforms which need to be orthogonal for separate radiating antenna elements, allows to choose reasonably the configuration of transmit and receive elements of MIMO antenna system and transmit waveforms.

Generalized ambiguity function is the normalized analog of generalized correlation integral of space-time signal processing for receive elements outputs of MIMO antenna system when point target returns are observed. The calculation and the comparison of generalized ambiguity functions in Cartesian and spherical coordinate systems are actual for linear array antennas consisting of transmit and receive elements. Such linear array antennas can represent two-dimensional antenna systems consisting of vertical or horizontal linear sub-arrays which can be replaced its phase center.

In this paper horizontal array consisted of 4 transmit and 8 receive elements located on the $x$-axis as the most simple example of MIMO antenna system is considered and the orthogonal transmit waveforms are the LFM waveforms with non-overlapping spectras. Classical spherical coordinate system is introduced as the first alternative to the Cartesian coordinate system and is formed by the Cartesian system $z$-axis which is normal to the $x$ axis of linear MIMO antenna array. The alternative is spherical coordinate system tagged referencing to Cartesian system $x$-axis. Two-dimensional cross-sections for considered spherical coordinate system alternatives can vary not only quantitatively but also qualitatively for the same geometric parameters of the MIMO antenna system and similar target location in the Cartesian coordinate system. In this paper we enounce the analysis of generalized ambiguity function cross-sections for linear MIMO antenna system and the choice of coordinate system which is the most suitable to the physical sense of the task.

\footnotetext{
*Corresponding author valch2008@yandex.ru
} 


\section{Generalized MIMO radar ambiguty fuction for the orthogonal LFM transmit waveforms}

Suppose that MIMO radar antenna system consists of $N_{t}+1$ transmit elements (T) and $N_{r}+1$ receive elements (R) with numbers $n=0, \ldots, N_{t}$ и $k=0, \ldots, N_{r}$. Transmit elements emit transmit signals with mutually orthogonal components on frequencies $\omega_{n}$ with step $\Delta \omega$ and complex envelope $\dot{A}(t)$ [3]:

$$
\dot{s}_{n}(t)=\dot{A}(t) \exp \left(j \omega_{n} t\right), \quad \omega_{n}=\omega_{0}+n \cdot \Delta \omega
$$

Suppose the complex envelope $\dot{A}(t)$ of the space-time signal (1) has bandwidth $\Delta \omega$. Along with this subpulses $\dot{s}_{n}(t)$ transmitted from element to element (at various $n$ ) are orthogonal subject to frequencies in (1), that is

$$
\int_{-\infty}^{\infty} \dot{s}_{n}(t) \dot{s}_{m}^{*}(t) d t=\int_{-\Delta \omega / 2}^{\Delta \omega / 2} \dot{G}\left(\omega-\omega_{n}\right) \dot{G}^{*}\left(\omega-\omega_{m}\right) d \omega=E \cdot \delta_{n, m}
$$

Where $\delta_{n, m}$ is the Kronecker's delta, $\dot{G}(\omega)$ is the Fourier spectrum of the comlpex envelope $\dot{A}(t), E$ is the waveform energy.

Suppose theat stationary (zero Doppler) point target has coordinate vector $\mathbf{R}$. Then after the echo observing of each transmitted signal the output of $k$-th receive element of MIMO antenna system with wide transmit beampattern is equal within constant factor:

$$
\dot{S}_{k}(t, \mathbf{R})=\sum_{n=0}^{N_{t}} \dot{A}\left(t-\tau_{n, k}(\mathbf{R})\right) \exp \left\{j \omega_{n}\left(t-\tau_{n, k}(\mathbf{R})\right)\right\}, k=0, \ldots, N_{r} .
$$

Bistatic delay is equal here

$$
\tau_{n, k}(\mathbf{R})=c^{-1}\left\{\left|\mathbf{R}-\mathbf{r}_{t n}\right|+\left|\mathbf{R}-\mathbf{r}_{r k}\right|\right\}
$$

where $c$ is the speed of electromagnetic wave propagation, $\mathbf{r}_{t n}$ and $\mathbf{r}_{r k}$ are the position of $n$ th transmitter and $k$-th receiver, defined in the same coordinate system of target position $\mathbf{R},|\mathbf{r}|$ is the magnitude symbol of a vector (the magnitude of a vector) $\mathbf{r}$.

The reference signal of STAP system corresponds to point target with any reference coordinate vector $\mathbf{R}_{0}$,

$$
\dot{S}_{k}\left(t, \mathbf{R}_{0}\right)=\sum_{n=0}^{N_{t}} \dot{A}\left(t-\tau_{n, k}\left(\mathbf{R}_{0}\right)\right) \exp \left\{j \omega_{n}\left(t-\tau_{n, k}\left(\mathbf{R}_{0}\right)\right)\right\}, k=0, \ldots, N_{r}
$$

and has the delay:

$$
\tau_{n, k}\left(\mathbf{R}_{0}\right)=\frac{1}{c}\left\{\left|\mathbf{R}_{0}-\mathbf{r}_{t n}\right|+\left|\mathbf{R}_{0}-\mathbf{r}_{r k}\right|\right\}
$$

The optimal STAP algorithm is based on the generalized correlation integral which is given in this case for point target by [2]: 


$$
\dot{Q}\left(\mathbf{R} ; \mathbf{R}_{0}\right)=\sum_{k=0}^{N_{r}} \int_{\infty}^{\infty} \dot{S}_{k}(t, \mathbf{R}) \dot{S}_{k}^{*}\left(t, \mathbf{R}_{0}\right) d t
$$

Substituting (3) and (6) to (7) gives with consideration to orthogonality of transmit signal sequence (1):

$$
\dot{Q}\left(\mathbf{R} ; \mathbf{R}_{0}\right)=\sum_{k=0}^{N_{r}} \sum_{n=0}^{N_{t}} \int_{\infty}^{\infty} \dot{A}\left(t-\tau_{n, k}(\mathbf{R})\right) \dot{A}^{*}\left(t-\tau_{n, k}\left(\mathbf{R}_{0}\right)\right) \exp \left\{j \omega_{n}\left[\tau_{n, k}\left(\mathbf{R}_{0}\right)-\tau_{n, k}(\mathbf{R})\right]\right\} d t,(8)
$$

where $*$ is the conjugate symbol. Replacing the complex envelopes for Fourier spectrum $\dot{G}(\omega)$

$$
\dot{A}(t)=\int_{-\infty}^{\infty} \dot{G}(\omega) \exp (j \omega t) d \omega
$$

allows to get expression for the generalized correlation integral through magnitude of the envelope spectrum:

$$
\dot{Q}\left(\mathbf{R} ; \mathbf{R}_{0}\right)=\sum_{k=0}^{N_{r}} \sum_{n=0}^{N_{t}} \int|\dot{G}(\omega)|^{2} \exp \left\{j\left(\omega+\omega_{n}\right)\left[\Delta \tau_{n, k}\left(\mathbf{R}, \mathbf{R}_{0}\right)\right]\right\} d \omega
$$

Delay increment is introduced here:

$$
\Delta \tau_{n, k}\left(\mathbf{R}, \mathbf{R}_{0}\right)=\tau_{n, k}\left(\mathbf{R}_{0}\right)-\tau_{n, k}(\mathbf{R}) .
$$

Integral over $d \omega$ in (9) is analytically computed for rectangular symmetrical envelope spectrum $\dot{G}(\omega)$ in the frequency band $[-\Delta \omega / 2, \Delta \omega / 2]$ with width being equal $\Delta \omega=2 \pi \Delta f$. Such model simulates the spectrum of transmit signal components with LFM exactly for high values of LFM subpulse time-bandwidth product $B$, which is equal to the product of subpulse width and frequency deviation, $B=T \Delta f>100 \ldots 200$. This assumption leads the expression (9) for generalized correlation integral:

$$
\dot{Q}\left(\mathbf{R} ; \mathbf{R}_{0}\right)=P_{A} \Delta \omega \sum_{k=0}^{N_{r}} \sum_{n=0}^{N_{t}} \operatorname{sinc}\left[\frac{\Delta \omega}{2 \pi} \cdot \Delta \tau_{n, k}\left(\mathbf{R}, \mathbf{R}_{0}\right)\right] \exp \left\{j \omega_{n}\left[\Delta \tau_{n, k}\left(\mathbf{R}, \mathbf{R}_{0}\right)\right]\right\},
$$

where $P_{A}=\left|\dot{G}_{A}(0)\right|, \operatorname{sinc}(x)=\frac{\sin (\pi x)}{\pi x}$.

Normalized generalized ambiguity function for MIMO radar is introduced on the basis of generalized correlation integral:

$$
\Psi\left(\mathbf{R}, \mathbf{R}_{0}\right)=\left|\dot{Q}\left(\mathbf{R}, \mathbf{R}_{0}\right)\right| /\left|\dot{Q}\left(\mathbf{R}_{0}, \mathbf{R}_{0}\right)\right| .
$$

Due to (12) we have the following calculating formula for generalized ambiguity function of MIMO radar antenna system with LFM transmit subpulses with high timebandwidth product: 


$$
\Psi\left(\mathbf{R}, \mathbf{R}_{0}\right)=\frac{1}{\left(N_{t}+1\right)\left(N_{r}+1\right)}\left|\sum_{k=0}^{N_{r}} \sum_{n=0}^{N_{t}} \operatorname{sinc}\left[\frac{\Delta \omega}{2} \Delta \tau_{n k}\left(\mathbf{R}, \mathbf{R}_{0}\right)\right] \exp \left\{j \omega_{n} \Delta \tau_{n k}\left(\mathbf{R}, \mathbf{R}_{0}\right)\right\}\right|
$$

This formula is truefor any spatial configuration of antenna system and allows to analyse the case of radiating of space-time transmit signals with orthogonal LFM sublulses with high time-bandwidth values. Special case of MIMO radar configuration with transmit and receive phase centers including the linear arrays and corresponding to azimuthal and elevation measurements can be considered for analysing coordinate system choice problem specified in the introduction.

\section{Two-dimensional generalized ambiguity function cross- sections comparison in different coordinate systems}

Figure 1 is the location of MIMO radar antenna system elements along $x$-axis in Cartesian coordinate system. Four transmit elements are shown in circles, eight receive elements are shown in rhomb figure. Distances between MIMO antenna system elements is chosen so that equivalent virtual receive/transmit array aperture is filled (dense), in this case, the step is $0.25 \lambda_{0}$, where $\lambda_{0}=10 \mathrm{sm}$ is the wavelength at center frequency of the first frequency component spectrum. Transmit elements emit LFM transmit waveforms with nonoverlapping spectrums. It is reached by their high time-bandwidth product.

The coordinates of the virtual array of $32=8 \times 4$ elements [1] are shown on the same figure 1 with triangles under the original MIMO array for convenience. There is a picture in meter scale.

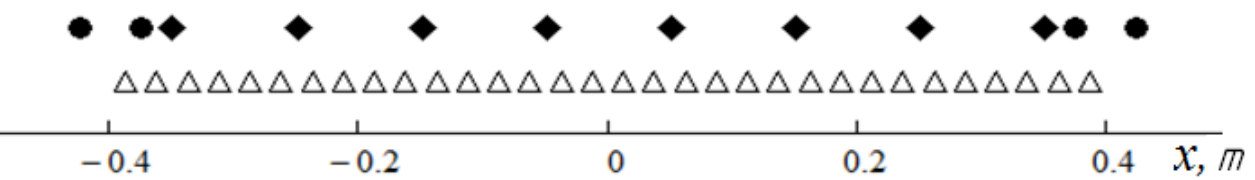

Fig. 1. The location of physical and virtual MIMO antenna system elements. Transmit elements are shown with dark circles, receive elements is shown with dark rhombs, virtual receive / transmit elements is shown with light triangles.

Normalized magnitude of generalized correlation integral is used as generalized ambiguity function (13).

Besides Cartesian coordinate vectors $\mathbf{r}=\|x, y, z\|$ and $\mathbf{r}_{0}=\left\|x_{0}, y_{0}, z_{0}\right\|$ two variants of spherical coordinates is outlined in the introduction to use further for generalized ambiguity function in accordance with transformations:

$$
\text { (I) }\left\{\begin{array} { c } 
{ x = r \operatorname { c o s } \varepsilon \cdot \operatorname { s i n } \beta } \\
{ y = r \operatorname { c o s } \varepsilon \cdot \operatorname { c o s } \beta } \\
{ z = r \operatorname { s i n } \varepsilon }
\end{array} \quad \text { (II) } \left\{\begin{array}{c}
x=r \sin \varepsilon \\
y=r \cos \varepsilon \cdot \cos \beta \\
z=r \cos \varepsilon \cdot \sin \beta
\end{array}\right.\right.
$$

Two right Cartesian coordinate systems satisfying this transformations is shown graphically in figures 2 , a, and 2, b, with corresponding angular and distance coordinates. The linear MIMO antenna system locates along $x$-axis in both variants, but azimuth $\beta$ is countered from different axes and elevation $\varepsilon$ is countered from different planes in (I) and (II) variants. 


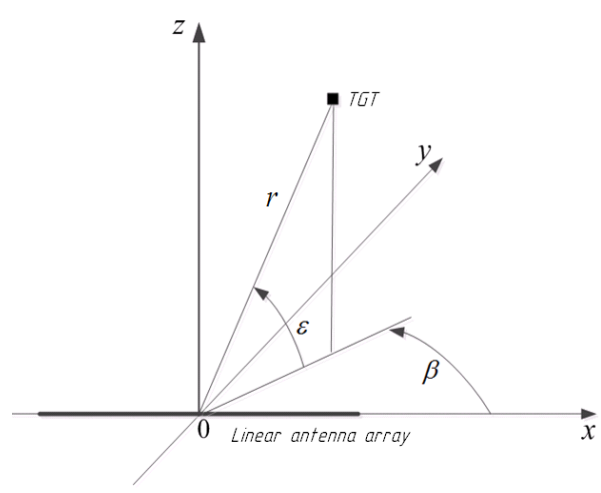

a

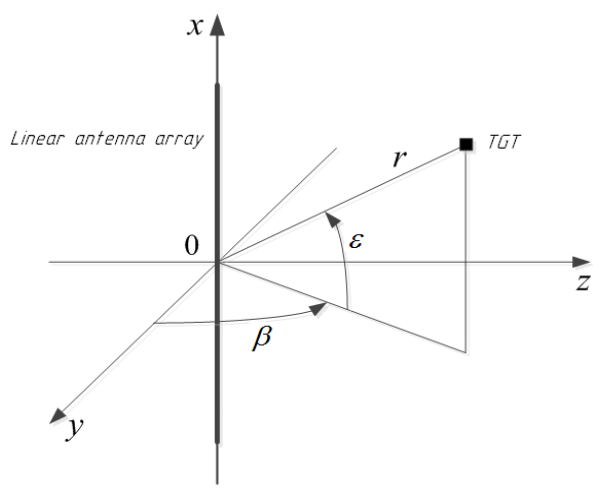

b

Fig. 2. Two variants of coordinate systems for generalized ambiguity function analyzing of MIMO antenna system. Coordinate system of type (I) is shown in (a), coordinate system of type (I) is shown in (b).

Two-dimensional generalized ambiguity function cross-sections examples are calculated with comparable scales and the same spatial target location and the same parameters of MIMO antenna system located along the $x$-axis in the fig. 1 and 2 . The Following transmit waveform parameters was were taken (accepted): full bandwidth of radiating is equal $\Delta F=\left(N_{t}+1\right) \Delta f=20 \mathrm{MHz}$ with a $\Delta f=5 \mathrm{MHz}$ frequency step and LFM sub-pulse bandwidth of each of four transmit element is and a $f_{0}=3 \mathrm{GHz}$ spectrum central frequency of the first LFM sub-pulse.

We will proceed from the definition of target location in spherical coordinates where $\mathbf{R}=\|r, \beta, \varepsilon\|$ for $\beta=40^{\circ}, \varepsilon=30^{\circ}, r=1000 \mathrm{M}$ for the identical analysis of generalized ambiguity function in Cartesian and spherical coordinates of (I) and (II) types. Generalized ambiguity function issue is logical to begin with drawing of its two-dimensional crosssections in Cartesian coordinates for plain earth surface. It is justified for short-range radars. The conversion of the spherical coordinates introduced higher to the Cartesian coordinate system leads to the following target rectangular coordinate vector $\mathbf{R}=\|x, y, z\|=\|663.4 \mathrm{M}, 500 \mathrm{M}, 556.7 \mathrm{M}\|$ for the (I) type transformation in (14), and $\mathbf{R}=\|x, y, z\|=\|556.7 \mathrm{M}, 500 \mathrm{M}, 663.4 \mathrm{M}\|$ for the (II) type transformation. Two-dimensional generalized ambiguity function cross-sections which respond to the first case are shown in fig. 3. The second case cross-sections are not shown here because its stay qualitatively the same. Its small quantitatively difference from the first case doesn't matter much of general generalized ambiguity function cross-sections issues in the Cartesian coordinate system.

This issue generally shows significant uncertainty in cross-sections for each of two coordinates in the $x z$-plane and $x y$-plane in fig. 3 , a, and 3, b. Total uncertainty is in crosssection for $(y, z)$ coordinates. Therefore Cartesian coordinates using is inexpediently for spatial target locating.

Two-dimensional generalized ambiguity function cross-sections - angular $(\beta, \varepsilon)$ and range-elevation $(r, \varepsilon)$ are shown in fig. 4 for spherical coordinates of (I) type and in fig. 5 for spherical coordinates of (II) type for target coordinates declared earlier. The comparison of obtained two-dimensional generalized ambiguity function cross-sections in two coordinate systems «range — azimuth — elevation» of (I) and (II) types (according to fig.2) showed follows. Significant uncertainty over true target coordinates is observed in 
the common coordinate system of (I) type in the «range - azimuth» « $\beta-\varepsilon »$ cross-section (fig. 4, a). Any uncertainty for elevation $\varepsilon$ is observed for local maximum near true target coordinates in the «range - elevation» $r-\varepsilon$ cross-section. Degree of this uncertainty depends on $\beta$ and grows with $\beta$ decrease, see fig. 6 , a, for $\beta=\varepsilon=10^{\circ}$.

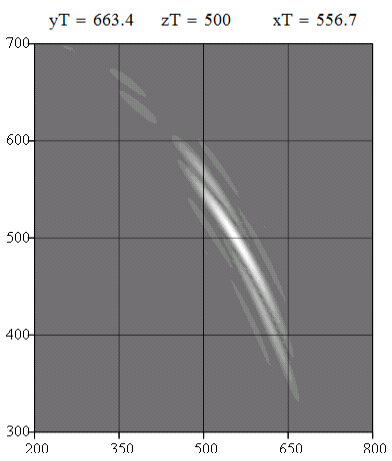

Qxz

a

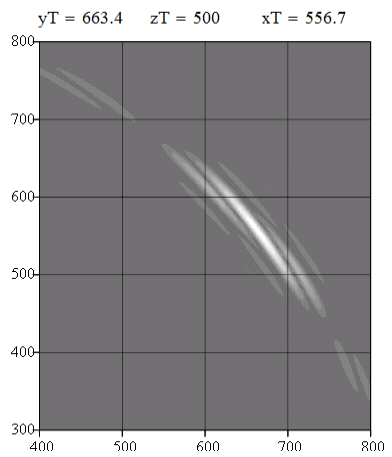

$\mathrm{b}$

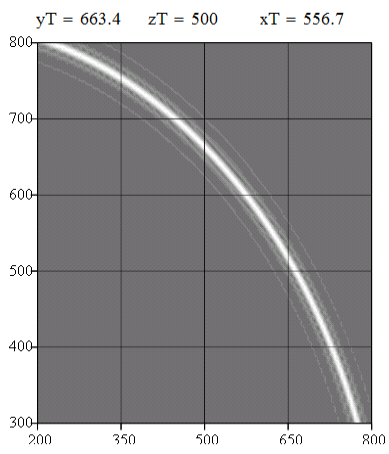

Qyz

C

Fig. 3. Example of two-dimensional generalized ambiguity function cross-sections in Cartesian coordinates: $X Z$-plane (a), $X Y$-plane (b), $Y Z$-plane (c).

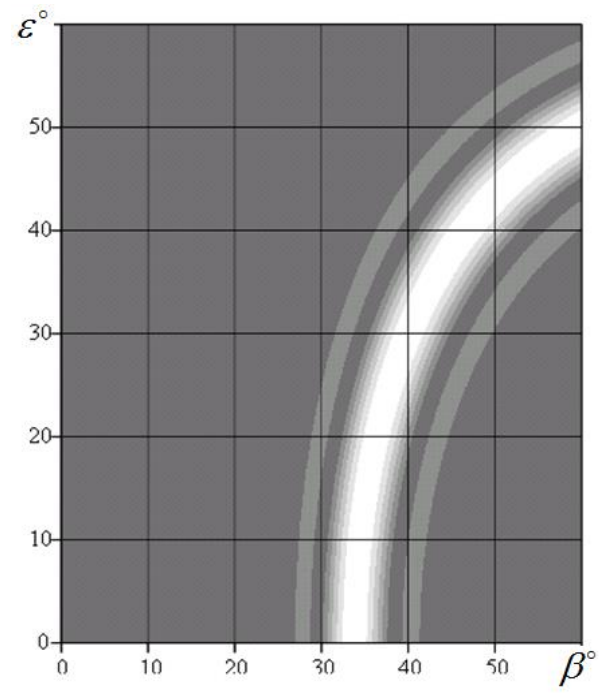

a

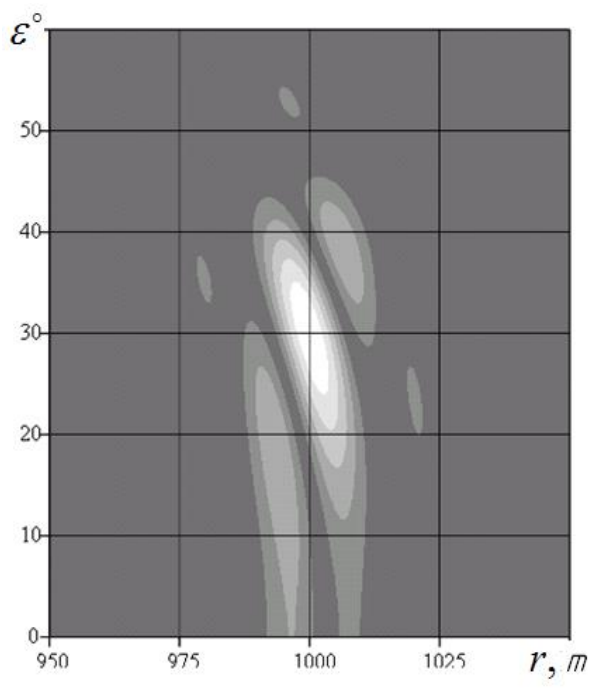

b

Fig. 4. Two-dimensional generalized ambiguity function cross-sections. «Azimuth - elevation» cross-section is shown in (a) and «range - elevation» cross-section is shown in (b) for (I) coordinate system type and target coordinates are $\beta=40^{\circ}, \varepsilon=30^{\circ}, r=1000 \mathrm{M}$.

Two-dimensional generalized ambiguity function $\beta-\varepsilon$ cross-section has evident physical sense in the modified coordinate system of II-type. This cross-section does not depend on azimuthal angle $\beta-\varepsilon$ by unambiguous maximum at the given elevation $\varepsilon=30^{\circ}$. Two-dimensional «range - elevation» $r-\varepsilon$ cross-section does not depend on azimuth $\beta$ and indicates the elevation correctly (fig. 5, b, 6, b). 


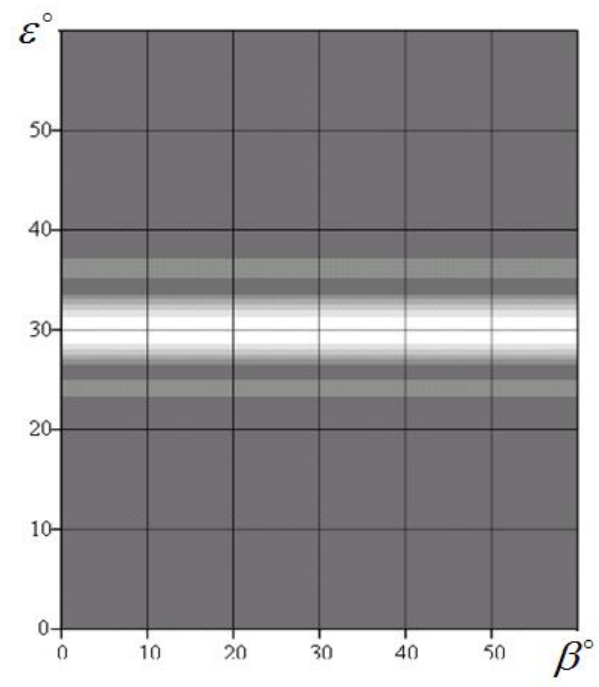

a

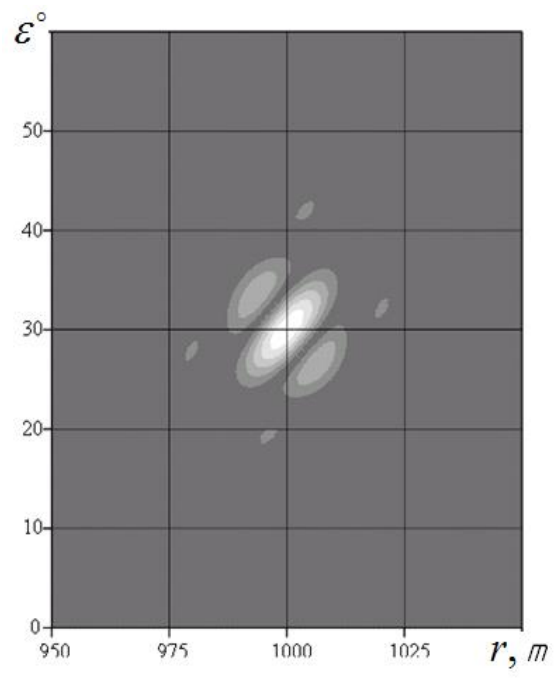

$\mathrm{b}$

Fig. 5. Two-dimensional generalized ambiguity function cross-sections. «Azimuth - elevation» cross-section is shown in (a) and «range - elevation» cross-section is shown in (b) for (II) coordinate system type and target coordinates are $\beta=40^{\circ}, \varepsilon=30^{\circ}, r=1000 \mathrm{M}$.

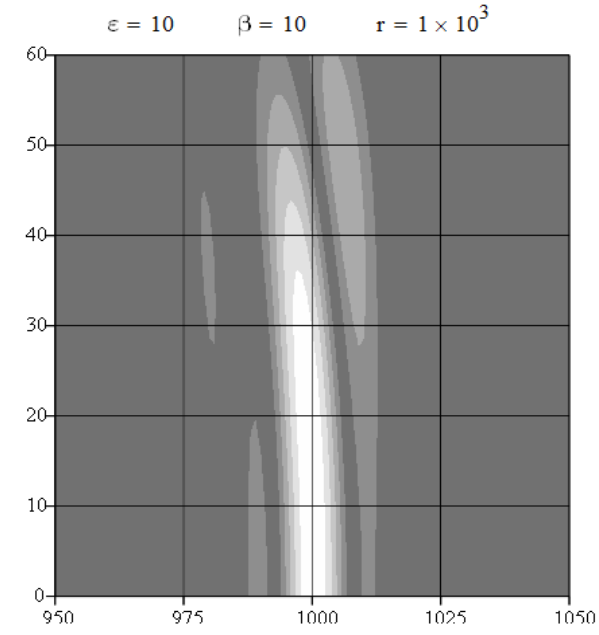

Qre

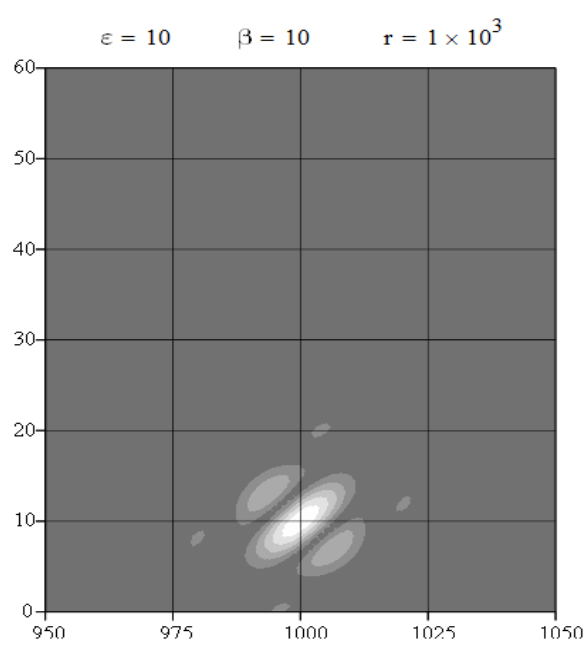

Qre1

a

$\mathrm{b}$

Fig. 6. Two-dimensional generalized ambiguity function «range - elevation» cross-sections for I-type (a) and II-type (b) coordinate system using for $\beta=10^{\circ}, \varepsilon=10^{\circ}, r=1000 \mathrm{M}$ target coordinates.

\section{Conclusion}

Generalized correlation integral of space-time signal processing at the output of receive elements of antenna system in MIMO radar with orthogonal LFM transmit waveforms radiation formulas are obtained. High time-bandwidth product approach is used for this waveforms. Two-dimensional generalized ambiguity function cross-sections proportional to 
generalized correlation integral magnitude are used in Cartesian and spherical coordinates for MIMO radar spatial resolution issue.

Generalized ambiguity function cross-section analysing showed main lobe width dependence on full frequency bandwidth $\Delta F$ of transmit waveforms of MIMO radar for all generalized ambiguity function two-dimensional cross-sections variants in Cartesian coordinates. This is explained by the relationship of all Cartesian coordinates $x, y, z$ with range $r$ which resolution $\delta r$ depends on full frequency bandwidth $\Delta F, \delta r=c /(2 \Delta F)$. In addition, Cartesian coordinate using leads to significant coordinate uncertainty. This reduces the improvement of spatial resolution due to increase of full frequency bandwidth of transmit waveforms.

Dependence on radiating frequency bandwidth is absent for generalized ambiguity function two-dimensional cross-sections for angular coordinates $(\beta, \varepsilon)$, in I-type and IItype spherical coordinates. Angular coordinates resolution and cross-section shape depend on the size of MIMO antenna system. Two-dimensional $(r, \varepsilon)$ and $(r, \beta)$ cross-section shape directly depends on range resolution $\delta r$ determinated frequency bandwidth and antenna system size. This dependence exists for spherical coordinates of I and II types. For linear MIMO antenna system it is necessary to choose physically suitable spherical coordinate system of II-type which does not lead to uncertainty in two-dimensional generalized ambiguity function cross-sections unlike coordinates of I-type. Such uncertainty for coordinates of I-type is in $(\beta, \varepsilon)$ plane and - in less degree - in $(r, \varepsilon)$ plane.

Given results and conclusions based on its show the significance of correct choice of coordinate system for analys of generalized ambiguity function and MIMO antenna system resolution including linear arrays consisting of transmit and receive phase centers. Introduced approach is important at the stage of resolution analysis and uncertainty of measurements in MIMO radar like in theoretical terms so radar specification design. This approach would be generalized for two-dimensional MIMO antenna systems with arbitary configuration.

\section{References}

1. J. Klare, O. Saalmann. MIRA-CLE X: A new Imaging MIMO-Radar for Multi-Purpose Applications. Proceedings of the 7th European Radar Conference. 30 September - 1 Paris, France. P. 129-132 (October 2010)

2. V. V. Chapursky. Izbrannye zadachu teorii sverkhshirokopolosnykh radiolokatsionnykh system. Izd. MGTU im. N.E. Baumana (Moscow, 2012)

3. I. V. Kryuchkov, V. V. Chapursky. Analytical approximation of the generalized ambiguity function of the MIMO synthetic-aperture radar system. Journal of communications technology and electronics, Vol. 62, № 11, p. 1133-1141 (2017) 